Pure Mathematical Sciences, Vol. 3, 2014, no. 1, 1 - 8

HIKARI Ltd, www.m-hikari.com

http://dx.doi.org/10.12988/pms.2014.3713

\title{
Admissible Wavelets on Groups and their Homogeneous Spaces
}

\author{
F. Esmaeelzadeh \\ Department of Mathematics, Bojnourd Branch \\ Islamic Azad University, Bojnourd, Iran \\ esmaeelzadeh@bojnourdiau.ac.ir \\ R. A. Kamyabi Gol \\ Department of Mathematics, Ferdowsi University of Mashhad \\ P. O. Box 1159, Mashhad 91775, Iran \\ kamyabi@ferdowsi.um.ac.ir
}

Copyright (c) 2014 F. Esmaeelzadeh and R. A. Kamyabi Gol. This is an open access article distributed under the Creative Commons Attribution License, which permits unrestricted use, distribution, and reproduction in any medium, provided the original work is properly cited.

\begin{abstract}
In this note, let $G$ be a locally compact group and $H$ be a compact subgroup of $G$. We investigate the square integrable representations of homogeneous spaces $G / H$ and admissible wavelets for these representations. Also, we consider the relation between the square integrable representations of locally compact groups and their homogeneous spaces. Moreover, the connection between existence of admissible wavelets for locally compact groups and their homogeneous spaces is described.
\end{abstract}

Mathematics Subject Classification: Primary 43A15; Secondary 43A85, $65 \mathrm{~T} 60$

Keywords: square integrable, admissible wavelet, homogeneous space, relatively invariant measure, strongly quasi invariant measure 


\section{Introduction and Preliminaries}

For a locally compact group $G$ with left Haar measure $\lambda$ it is well known that a continuous unitary representation $\pi$ of $G$ is called square integrable if there exists a non-zero vector $\zeta$ in Hilbert space $\mathcal{H}$ such that

$$
\int_{G}|<\pi(x) \zeta, \zeta>|^{2} d \lambda(x)<\infty
$$

Such a unital vector $\zeta$ is called an admissible vector. The square integrable representations on homogeneous spaces that admit $G$-invariant measure and relatively invariant measure have been studied in $[1,5]$. In this manuscript we investigate the relation between square integrable representations of locally compact group $G$ and its homogeneous space $G / H$, in which $H$ is compact subgroup of $G$. To be more precise we need to fix some notations and review some basic concepts. For more details one may refer to $[8,6,12,3]$.

Let $G$ be a locally compact group and $H$ be a closed subgroup of $G$ with left Haar measures $\lambda$ and $\lambda_{H}$, respectively. Consider $G / H$ as a homogeneous space on which $G$ acts from left and the Radon measure $\mu$ on $G / H$ is said to be $G$-invariant if $\mu_{x}(y H)=\mu(y H)$ for all $x, y \in G$, where $\mu_{x}$ is defined by $\mu_{x}(E)=\mu(x E)$ (for Borel subset $E$ of $\left.G / H\right)$. It is well known that there is a $G$-invariant Radon measure $\mu$ on $G / H$ if and only if $\left.\Delta_{G}\right|_{H}=\Delta_{H}$, where $\Delta_{G}, \Delta_{H}$ are the modular functions on $G$ and $H$, respectively. In this case we have,

$$
\int_{G} f(x) d \lambda(x)=\int_{G / H} P f(x H) d \mu(x H)=\int_{G / H} \int_{H} f(x h) d \lambda_{H}(h) d \mu(x H),
$$

in which $P f(x H)=\int_{H} f(x h) d \lambda_{H}(h)$ is surjective continuous map from $C_{c}(G)$ onto $C_{c}(G / H)$.

The Radon measure $\mu$ on $G / H$ is called strongly quasi invariant if there is a positive continuous function $\alpha$ of $G \times G / H$ such that $d \mu_{x}(y H)=\alpha(x, y H) d \mu(y H)$, for all $x, y \in G$. If the function $\alpha(x,$.$) reduce to constants, the measure \mu$ is called relatively invariant measure.

A rho-function for the pair $(G, H)$ is defined to be a continuous positive function $\rho$ from $G$ which satisfies

$$
\rho(x h)=\frac{\Delta_{H}(h)}{\Delta_{G}(h)} \rho(x) \quad(x \in G, h \in H) .
$$

In [10] it has been shown that the existence of a homomorphism rho-function for the pair $(G, H)$ is a necessary and sufficient condition for the existence of 
a relatively invariant measure $\mu$ on $G / H$.

In this paper we introduce square integrable representations of homogeneous space $G / H$ equiped with a relatively invariant measure $\mu$. The main aim in this paper is to compare square integrable representations of locally compact groups and their homogeneous spaces. Finally we describe the relation between admissible wavelets on groups and their homogeneous spaces.

\section{Main Result}

Let $G$ be a locally compact group and $\mathcal{H}$ be a Hilbert space. We recall square integrable representations of locally compact groups.

An irreducible representation $\pi$ of group $G$ is said to be square integrable if there exists a nonzero element $\zeta$ in $\mathcal{H}$ such that

$$
\int_{G}|<\zeta, \pi(x) \zeta>|^{2} d \lambda(x)<\infty
$$

The condition (2.1) is known as the admissibility condition for the square integrable representations of $G$ on $\mathcal{H}$. The element $\zeta \in \mathcal{H}$ for which $\|\zeta\|=1$ is called admissible wavelet for the square integrable representation $\pi$ of group $G$. The wavelet constant associated to the admissible wavelet is denoted by $c_{\zeta}$ and defined as:

$$
c_{\zeta}=\int_{G}|<\zeta, \pi(x) \zeta>|^{2} d \lambda(x) .
$$

( For more details about admissible wavelets on locally compact groups the reader can be consult with $[13,9])$.

In [5], we have studied the squre integrable representations of homogeneous spaces and admissible wavelets for these representations. Here we define representations of homogeneous spaces which will be needed in the difinition of square integrable representations of homogeneous spaces.

$A$ unitary representation of the homogeneous space $G / H$ is a map $\varpi$ from $G / H$ into the group $U(\mathcal{H})$, of all unitary operators on some nonzero Hilbert space $\mathcal{H}$, for which the map $x H \mapsto<\varpi(x H) \zeta, \xi>$ from $G / H$ into $\mathbb{C}$ is continuous, for each $\zeta, \xi \in \mathcal{H}$ and

$$
\varpi(x y H)=\varpi(x H) \varpi(y H), \quad \varpi\left(x^{-1} H\right)=\varpi(x H)^{*},
$$

for each $x, y \in G$. It is worthwhile to note that this defines a unitary representation $\pi$ of $G$ in which the subgroup $H$ is contained in the kernel of $\pi$. Conversely, any unitary representation $\pi$ of $G$ which is trivial on $H$ induces a unitary representation $\varpi$ of $G / H$, by letting $\varpi(x H)=\pi(x)$. Moreover, a closed subspace 
$\mathcal{M}$ of $\mathcal{H}$ is said to be invariant with respect to $\varpi$ if $\varpi(x H) \mathcal{M} \subseteq \mathcal{M}$, for all $x \in G$. A unitary representation $\varpi$ is said to be irreducible if the only invariant subspaces of $\mathcal{H}$ are $\{0\}$ and $\mathcal{H}$.

An irreducible representation $\varpi$ of $G / H$ on $\mathcal{H}$, in which $G$ is a locally compact group and $H$ is a compact subgroup of $G$, is said to be square integrable if there exists a nonzero element $\zeta \in \mathcal{H}$ such that

$$
\int_{G / H} \frac{\rho(e)}{\rho(x)}|<\zeta, \varpi(x H) \zeta>|^{2} d \mu(x H)<\infty,
$$

where $\mu$ is a relatively invariant measure on $G / H$ which arises from a rho function $\rho: G \rightarrow(0, \infty)$. If $\zeta$ satisfies (2.2), it is called an admissible vector. An admissible vector $\zeta \in \mathcal{H}$ is called an admissible wavelet if $\|\zeta\|=1$. In this case, we define the wavelet constant $c_{\zeta}$ as

$$
c_{\zeta}:=\int_{G / H} \frac{\rho(e)}{\rho(x)}|<\zeta, \varpi(x H) \zeta>|^{2} d \mu(x H) .
$$

We call $c_{\zeta}$ the wavelet constant associated to the admissible wavelet $\zeta$. Note that since $H$ is compact subset of $G$, the definition (2.2) is well define.

In the sequle we intend to establish a relation between square integrable representations of locally compact groups and their homogeneous spaces. We recall that a unitary representation of homogenous space $G / H$ is in one to one correspondence to a unitary representation of $G$ whose kernel $\pi$, which is denoted by $N$, contains $H$. We denote by $q_{N}, q_{H}, p$, the canonical mappings of $G$ onto $G / N$, of $G$ onto $G / H$ and of $N$ onto $N / H$. Let $\lambda_{N}$ and $\lambda_{H}$ be the left Haar measure on $N$ and $H$, respectively. Then there exists a $G$-invariant measure $\mu_{N / H}$ on $N / H$. On the otherhand, let $\lambda$ be a left Haar measure on $G$. One can then form a left invariant mesures $\mu_{G / N}$ on quotient group $G / N$ and a relatively invarint measure $\mu_{G / H}$ on homogeneous space $G / H$ which arises from rho-function $\rho$ of $G$. It is clear that, the mapping $(x, n) \mapsto q_{H}(x n)$ of $G \times N$ into $G / H$ is continuous. Since $q_{H}(x n h)=q_{H}(x n)$, for all $h \in H$, this mapping defines a continuous mapping of $G \times(N / H)$ into $G / H$. Whence for each fixed $x \in G$, the mapping $\psi_{x}$ of $N$ into $G$ such that $\psi_{x}(n)=x n$, define a mapping $\omega_{x}$ of $N / H$ into $G / H$ in which

$$
\omega_{x}(p(n))=q_{H}\left(\psi_{x}(n)\right)=q_{H}(x n) .
$$

It is easy to show that $\psi_{x n}=\psi_{x} O \varrho_{N}(n)$, thererfore that $\omega_{x n}=\omega_{x} O \varrho_{N / H}(n)$, for all $n \in N$, in which $\varrho_{N}(n)\left(n^{\prime}\right)=n n^{\prime}$. The following lemma shows that the map $\omega_{x}$ is proper. 
Lemma 2.1. Let $E$ be a compact subset of $G / H$ and $K$ be a compact subset of $G$. Then $\cup_{x \in K} \omega_{x}^{-1}(E)$ is relatively compact in $N / H$. In particular, $\cup_{x \in K} \omega_{x}^{-1}(E)$ is contained in a compact subset of $N / H$.

Proof. Let $F$ be a compact subset of $G$ such that $q_{H}(F)=E$. Let $L$ be the set of $n \in N$ such that $K n$ intersects $F$. Then $L$ is compact (see [2], chapterIII, $\oint 4.5$, theorem1). Let $n \in N$, such that $p(n) \in \cup_{x \in K} \omega_{x}^{-1}(E)$. Thus there exists $x \in K$ such that $\omega_{x}(p(n)) \in E$. i.e. $q_{H}(x n) \in E$ and since $q_{H}(F)=$ $E$, there exists $h \in H, x n h \in F$. Then $n h \in L$. So $p(n h)=p(n) \in p(L)$. That is $\cup_{x \in K} \omega_{x}^{-1}(E) \subseteq p(L)$.

Let $\mathcal{M}(N / H)$ and $\mathcal{M}(G / H)$ be complex measure spaces on homogeneous spaces $N / H$ and $G / H$, respectively, as introduced in $[3,12]$. Lemma 2.2 shows that the mapping $\omega_{x}$ is proper. Then $\omega_{x}$ extends continuously to a map from $\mathcal{M}(N / H)$ into $\mathcal{M}(G / H)\left([7]\right.$, Section 4.5). Now let $\varphi \in C_{c}(G / H)$. Define the function $\Psi$ of $G$ into $\mathcal{M}(G / H)$ such that

$$
\Psi(x)=<\varphi, \omega_{x}\left(\mu_{N / H}\right)>:=\int_{N / H} \varphi\left(\omega_{x}(p(n)) d \mu_{N / H}(p(n)) .\right.
$$

The function $\Psi$ is continuous and compact support. Morevere by $G$-invariant measure $\mu_{N / H}$, we have

$$
\begin{aligned}
\Psi(x n) & =<\varphi, \omega_{x n}\left(\mu_{N / H}\right)> \\
& =<\varphi, \omega_{x} O \varrho_{N / H}(n)\left(\mu_{N / H}\right)> \\
& =\int_{N / H} \varphi\left(\omega_{x} o \varrho_{N / H}(n)\left(p\left(n^{\prime}\right)\right)\right) d \mu_{N / H}\left(p\left(n^{\prime}\right)\right) \\
& =\int_{N / H} \varphi\left(\omega_{x}\left(n n^{\prime} H\right)\right) d \mu_{N / H}\left(p\left(n^{\prime}\right)\right) \\
& =\int_{N / H} \varphi\left(\omega_{x}\left(n^{\prime} H\right)\right) d \mu_{N / H}\left(p\left(n^{\prime}\right)\right) \\
& =\varphi\left(\omega_{x}\left(\mu_{N / H}\right)\right) \\
& =\Psi(x),
\end{aligned}
$$

for $n \in N$. Then the mapping $\tilde{\Psi}$ of $G / N$ into $\mathcal{M}(G / H)$ in which

$$
\tilde{\Psi}\left(q_{N}(x)\right)=<\varphi, \omega_{x}\left(\mu_{N / H}\right)>,
$$

is continuous with compact support, for all $\varphi \in C_{c}(G / H)$.

Proposition 2.2. Let $\varphi \in C_{c}(G / H)$. Then

$$
\int_{G / N}<\varphi, \omega_{x}\left(\mu_{N / H}\right)>d \mu_{G / N}\left(q_{N}(x)\right)=\int_{G / H} \varphi\left(q_{H}(x)\right) d \mu_{G / H}\left(q_{H}(x)\right) .
$$

Proof. By (1.1), for $\varphi \in C_{c}(G / H)$ we have

$$
\int_{G / H} \varphi\left(q_{H}(x)\right) d \mu_{G / H}\left(q_{H}(x)\right)=\int_{G} f(x) d \lambda(x),
$$


where $\varphi=P f$ and $f \in C_{c}(G)$. Also,

$$
\begin{aligned}
& \int_{G / N}<\varphi, \omega_{x}\left(\mu_{N / H}\right)>d \mu_{G / N}\left(q_{N}(x)\right)= \\
& \int_{G / N} \int_{N / H}\left(\operatorname { P f } \left(\omega_{x}(p(n)) d \mu_{N / H}(p(n)) d \mu_{G / N}\left(q_{N}(x)\right)=\right.\right. \\
& \int_{G / N} \int_{N / H}\left(\operatorname{Pf}\left(q_{H}(x n)\right) d \mu_{N / H}(p(n)) d \mu_{G / N}\left(q_{N}(x)\right)=\right. \\
& \int_{G / N} \int_{N / H} \int_{H} R_{x} f(n h) d \lambda_{H}(h) d \mu_{N / H}(p(n)) d \mu_{G / N}\left(q_{N}(x)\right)= \\
& \int_{G / N} \int_{N} f(x n) d \lambda_{N}(n) d \mu_{G / N}\left(q_{N}(x)\right)= \\
& \int_{G} f(x) d \lambda(x) .
\end{aligned}
$$

Corollary 2.3. (i)) Let $\varphi$ be a $\mu_{G / H}$-integrable function on $G / H$. There exists a $\mu_{G / N}$-negligible subset $E$ of $G / N$ having the following property: if $x \in$ $G$ is such that $q_{N}(x) \notin E$, then the function $\varphi \omega_{x}$ on $N / H$ is $\mu_{N / H}$-integrable. The integral $\int_{N / H} \varphi\left(\omega_{x}(p(n)) d \mu_{N / H}\left(p(n)\right.\right.$ is a $\mu_{G / N}$-integrable function and

$$
\int_{G / H} \varphi\left(q_{H}(x)\right) d \mu_{G / H}\left(q_{H}(x)\right)=\int_{G / N} d \mu_{G / N}\left(q_{N}(x)\right) \int_{N / H} \varphi\left(\omega_{x}(p(n)) d \mu_{N / H}(p(n)) .\right.
$$

(ii) suppose that there exists a bounded positive measure $\mu_{G / H}$ on homogenous space $G / H$. Then there exists a bounded positive measure on homogeneous space $N / H$.

Proof. (i) By proposition 2.2 we have,

$$
\begin{aligned}
\int_{G / H} \varphi\left(q_{H}(x)\right) d \mu_{G / H}\left(q_{H}(x)\right) & =\int_{G / N}<\varphi, \omega_{x}\left(\mu_{N / H}\right)>d \mu_{G / N}\left(q_{N}(x)\right) \\
& =\int_{G / N} \int_{N / H}\left(\varphi \left(\omega_{x}(p(n)) d \mu_{N / H}(p(n)) d \mu_{G / N}\left(q_{N}(x)\right) .\right.\right.
\end{aligned}
$$

(ii) The function 1 on $G / H$ is $\mu_{G / H^{-}}$integrable. By the part (i), the function 1 on $N / H$ is $\mu_{N / H}$-integrable. Thus $\mu_{N / H}$ is bounded.

As before we mention, a unitary representation $\varpi$ of homogeneous space $G / H$ define a representation $\pi$ of $G$ such that $k e r \pi=N$ contains $H$. Consider $\tilde{\pi}$, the representation of $G / N$ by letting $\tilde{\pi}(x N)=\pi(x)$. The following theorem show that the representation $\varpi$ of $G / H$ is square integrable if and only if the representation $\tilde{\pi}$ of quotient group $G / N$ is square integrable.

Theorem 2.4. Let $\varpi$ be a unitary representation of $G / H$. The unitary representation $\varpi$ of $G / H$ is square integrable if and only if the representation $\tilde{\pi}$ of quotient group $G / N$ is square integrable. 
Proof. Assume that the representation $\varpi$ is an irreducible. Let $\pi$ the associated representation of $G$ and let $N$ be the kernel of representation $\pi$. The representation $\varpi$ is irreducible if and only if $\pi$ is irreducible or equivalently, if the representation $\tilde{\pi}$ is irreducible. Then, by the corollary 2.3 and since the $G$-invariant measure $\mu_{N / H}$ is finite [11], we have

$$
\int_{G / H} \varphi(x H) d \mu_{G / H}(x H)=\mu(N / H) \int_{G / N} \varphi(x N) d \mu_{G / N}(x N) .
$$

Here $\varphi(x H)=\mid\left\langle\varpi(x H) \zeta, \zeta>\left.\right|^{2}\right.$. So, the representation $\varpi$ is a square integrable of $G / H$ if and only if $\pi$ induce a representation $\tilde{\pi}$ of quotient group $G / N$ where $\tilde{\pi}$ is square integrable.

We can conclude that there exsits an admissible wavelet for representation $\varpi$ of homogeneous space $G / H$ if and only if there exists an admissible wavelet for induced representation $\tilde{\pi}$ of quotient group $G / N$.

Corollary 2.5. A unital vector $\zeta$ in Hilbert space $\mathcal{H}$ is an admissible wavelet for representation $\varpi$ of homogeneous space $G / H$ if and only if $\zeta$ in $\mathcal{H}$ is an admissible wavelet for representation $\tilde{\pi}$ of qoutient group $G / N$.

The following example support our tecnical considerations in this note.

Example 2.6. Consider Euclidean group $G=S O(n) \times_{\tau} \mathbb{R}^{n}$ with group operations

$$
\left(R_{1}, p_{1}\right) \cdot\left(R_{2}, p_{2}\right)=\left(R_{1} R_{2}, R_{1} p_{2}+p_{1}\right), \quad(R, p)^{-1}=\left(R^{-1},-R^{-1} p\right) .
$$

Put $n=2$ in $G$, i.e. $G=S O(2) \times{ }_{\tau} \mathbb{R}^{2}$ and $\mathcal{H}=L^{2}\left(S^{1}\right) \simeq L^{2}[-\pi, \pi]$. In this setting any $R \in S O(2)$ and $s \in S^{1}$ are given explicitly by

$$
\begin{gathered}
R=\left(\begin{array}{cc}
\cos \theta & \sin \theta \\
-\sin \theta & \cos \theta
\end{array}\right) \\
s=\left(\begin{array}{c}
\sin \gamma \\
\cos \gamma
\end{array}\right)
\end{gathered}
$$

The representation $\pi$ on $G$, is defined as

$$
\pi\left(\theta, p_{1}, p_{2}\right) \psi(\gamma)=e^{i\left(p_{1} \sin \gamma+p_{2} \cos \gamma\right.} \psi(\gamma-\theta),
$$

for all $\left(\theta, p_{1}, p_{2}\right) \in G, \psi \in L^{2}\left(S^{1}\right)$. Since this representation of $G$ is not square integrable [4], we are looking for suitable representation of its homogeneous space. Consider $H=\left\{\left(0,0, p_{2}\right) \in G\right\}$. Thus the representation of $G / H$ is square integrable [5]. By theorem 2.4 the representation $\varpi$ of homogeneous space $G / H$ is square integrable if and only if the representation $\tilde{\pi}$ of qoutient group $G / N$ is square integrable, where $N=\operatorname{ker} \pi=\left\{\left(0,-p_{2} \operatorname{cotg} \gamma, p_{2}\right), p_{2} \in\right.$ $\mathbb{R}\}$. 


\section{References}

[1] S.T. Ali, J-P. Antoine, P. Vandergheynst, R. Murenzi, Two Dimensional Wavelets and their Relatives, Springer-Verlag, New York, 2004.

[2] N. Bourbaki, Elements of Mathematics, General Topology, springer, Verlag Berlin, New york,1971.

[3] N. Bourbaki, Elements of Mathematics, Intrgration, springer, Verlag Berlin, New york, 1965.

[4] S. Dahlke, G. Steidl, G. Teschke Weighted coorbit spaces and Banach frames on homogeneous spaces, J. Fourier Anal, Appl. 10 (2004), no. 5, 507-539.

[5] F. Esmaeelzadeh, R.A. Kamyabi Gol, R. Raisi Tousi, On the continuous wavelet transform on homogeneous spases,Int. J. Wavelets. Multiresolut, Vol 10, No4, 2012.

[6] J.M.G. Fell, R.S. Doran, Representation Of *-algebras, Locally Compact Groups, and Banach ${ }^{*}$-algebraic Bundles, Vol 1, Academic Press, 1988.

[7] G.B. Folland, Real Analysis, CRC Press, 1999.

[8] G.B. Folland, A Course in Abstract Harmonic Analysis, CRC Press, 1995.

[9] H. Führ, Abstract Harmonic Analysis of Continuous Wavelet Transform, Springer Lecture Notes in Mathematics, Nr. 1863, Berlin, 2005.

[10] R.A. Kamyabi Gol, N. Tavallaei, convolution and homogeneous spaces. Bull. Iranian Math. Soc. 35(2009), no.1, 129-146, 281.

[11] G. D. Mostow , homogeneous spaces with invariant measure.Annals of Mathematics. Second Series, Vol. 75, No. 1 (Jan., 1962), pp. 17-37

[12] H. Reiter, J. Stegeman, Classical Harmonic Analysis and Locally compact Group, claredon press, 2000.

[13] M.W. Wong, Wavelet Transform and Localization Operators. Birkhauser Verlag, Basel-Boston-Berlin, 2002.

Received: July 1, 2013 